\title{
Análisis psicométrico de la Prueba de Sentido de Vida en escolares de la ciudad de Arequipa (Perú)
}

\author{
Psychometrical analysis of Purpose in Life Test in school students from \\ Arequipa City (Peru)
}

\author{
Julio Cesar Huamani Cahua ${ }^{1}$ \\ Walter Lizandro Arias Gallegos ${ }^{2}$ \\ Universidad Católica San Pablo. Arequipa, Perú
}

Recibido: $27-03-18$

Aceptado: $23-07-18$

\begin{abstract}
Resumen
En este artículo se presenta el análisis psicométrico de la Prueba de Sentido de Vida de Crumbaughy Maholick (1969) en una muestra de escolares de la ciudad de Arequipa (Perú). Para ello, se seleccionó una muestra no probabilística de 872 estudiantes de 4to y 5 to de secundaria de cinco instituciones educativas públicas de la ciudad, a quienes se les aplicó la versión española del Purpose in Life Test (Noblejas, 1994). Se analizaron la validez de constructo y la confiabilidad de la prueba a través del análisis factorial exploratorio y confirmatorio, y la prueba alfa de Cronbach. Los resultados confirmaron la estructura de cuatro factores de Noblejas (2000), pero se eliminan los ítems 4 y 19 y se tienen índices adecuados de confiabilidad que superan el 0.7 para la puntuación total y cada uno de los factores.

Palabras clave: Sentido de vida; análisis existencial; adolescentes; confiabilidad; análisis fac-
\end{abstract} torial confirmatorio.

\begin{abstract}
In this article, we present the psychometrical analysis of the Purpose in Life Test ofCrumbaugh y Maholick (1969) in a sample of high school students from Arequipa City (Peru). We selected a non-probabilistic sample of 872 students of 4th and 5th grade of five public high schoolsinto the city, who were tested by the Spanish version of Purpose in Life Test (Noblejas, 1994). We analyze the construct validity and reliability of the test through the exploratory and confirmatory factor analysisand the Cronbach's Alfa Test. The results confirm the four factors structure reported by Noblejas (2000), but the items 4 and 19 were eliminated and the reliability indexes are higher than 0.7 for the total punctuationand each factor.
\end{abstract}

Keywords: Purpose in life; existential analysis; adolescents; reliability; confirmatory factor analysis.

\footnotetext{
1 Docente Universidad Católica San Pablo. Autor para correspondencia. Email: jchuamani@ucsp.edu.pe 2 Docente Universidad Católica San Pablo. Email: walterlizandro@hotmail.com

(C) Los autores. Este artículo es publicado por la Revista de Investigación en Psicología de la Facultad de Psicología, Universidad Nacional Mayor de San Marcos. Este es un artículo de acceso abierto, distribuido bajo los términos de la licencia Creative Commons Atribucion - No Comercia_Compartir Igual 4.0 Internacional. (http://creativecommons.org/licenses/by-nc-sa/4.0/) que permite el uso no comercial, distribución y reproducción en cualquier medio, siempre que la obra original sea debidamente citada.
} 
El sentido de vida ha sido un concepto desarrollado principalmente por Viktor Frankl (1905-1997), neurólogo y psiquiatra austriaco que luego de ser tomado prisionero y recluido en dos campos de concentración nazi, propuso la logoterapia como tercera escuela vienesa de psicoterapia (Pareja, 2007). El libro "El hombre en busca de sentido" narra las experiencias de Frankl en los campos de concentración de Auschwitz y Dachau como el prisionero 119.104 desde 1942 hasta 1945 (Frankl, 1983). Estas experiencias le llevaron a plantear la importancia de la búsqueda de sentido en la vida del hombre como un asunto trascendente para su correcto ajuste psicológico (Frankl,1962/ 2002). De acuerdo con Frankl (1965/1987), cuando el hombre encuentra sentido a su vida es capaz de soportar diversas adversidades por muy dolorosas que sean, porque responden un propósito personal e íntimo que le permite trascender. Cuando el hombre no encuentra sentido a su vida o a lo que hace, cae en un vacío existencial que es causa de una neurosis noógena (Frankl, 1980). De este modo, Frankl (1994) desarrolla la logoterapia como un método basado en el análisis existencial, para dotar de sentido el mundo interno de la persona.

La palabra "logoterapia", que surge de la conjunción de los términos griegos logos (sentido, significado o propósito) y therapeia (curación o tratamiento), podría definirse como la terapia basada en la búsqueda de sentido. Sin embargo, Yalom (1980) señala muy acertadamente, que debe distinguirseentre "significado" y "propósito", pues mientras el significado hace alusión a la coherencia, el propósito refiere un objetivo y una intención en el comportamiento humano. Para Frankl (1946/1983) el sentido de vida se define como el para qué, el motivo, la razón que nos impulsa a lograr algo, o para ser de una manera determinada.

En tal sentido, aunque la búsqueda de un "logos" puede rastrearse desde la época de los griegos (Rey de Castro, 2011), no toda búsqueda de sentido responde al modelo que plantea la logoterapia, pues la palabra sentido tiene un origen polisémico, que ha sido usado en varias escuelas psicológicas. La escuela dialéctico materialista le define como un "criterio o noción interior del sujeto en sí, sustentada en las tradiciones individuales, concepciones, propósitos, utopías, ideas y creencias" (Jiménez, 2004, p. 130). Por otro lado, desde diversas escuelas psicológicas se ha puesto énfasis en el autoconocimiento que es la base del sentido de vida (García, 1999). William James, padre del funcionalismo americano, en "Principios de Psicología" también señala que diversos aspectos de la autoconciencia que desembocan en el sentido de identidad (James, 1890/1996), mientras que Alfred Adler (1914/1961) introdujo el concepto de "estilo de vida"como una vía a la superación de la neurosis. Desde un enfoque más cognitivo, autores como George Kelly (1955/1966) y Albert Ellis (1962/1980) han conferido capital importancia a las nociones de "constructos personales" y "pensamientos irracionales", respectivamente, como notables influencias del sentido que se le otorgan a las experiencias vividas. 
Por otro lado, el miedo existencial al "no ser" también ha sido resaltado por Jaspers (1913/1996) como una vía para el desarrollo de la neurosis, y al igual que Frankl, resalta que el sufrimiento puede aportar sentido a la vida, pero lo que cuenta es la actitud que se asume para afrontar el dolor (Pinedo, 2014). Asimismo, la negación del dolor alude a una concepción de la vida, del hombre y de Dios; que Frankl (1985/2006) entiende como una puerta abierta hacia la neurosis. Por ello, diversos estudios han reportado que las personas religiosas presentan mayor sentido de vida, mayor bienestar psicológico y menor problemas de salud (Quiceno y Vinaccia, 2009).

Ahora bien, el sentido de vida, como constructo psicológico, ha generado una gran cantidad de investigación que sobre la base de las ideas de Frankl,le han vinculado con una diversidad de desórdenes mentales, dado su arraigo en la noción de vacío existencial. De este modo, la falta de sentido en la vida y el vacío existencial han sido considerados como factores causales del suicidio y la depresión, mediados por ciertos factores psicosociales, en los jóvenes y los adolescentes (Ceballos, y Chávez, 2016). En el estudio de Medina, Herazo, Barrios, Rodelo y Salinas (2017), por ejemplo, se tomó una muestra de 876 estudiantes de ambos sexos de una institución educativa de Colombia, determinándose que la falta de apoyo familiar, la impulsividad y los sentimientos de depresión se asociaban a un alto índice de desesperanza que motivó ideas e intentos de suicidio, en más de la mitad de la muestra.En tal sentido, se ha probado que aquellas personas con altos niveles de vacío existencial también presentan puntuaciones más altas en la variable desesperanza (García-Alandete, Gallego-Pérez y Pérez-Delgado, 2009; Gallego y García, 2004).

Así pues, los jóvenes con un sentido de vida poco desarrollado, presentan inadecuados mecanismos de afrontamiento del estrés y se encuentran más propensos a incurrir en conductas suicidas (Edwards y Holden, 2003). De hecho, las tasas de suicidio en jóvenes y adolescentes, han experimentado un incremento global en los últimos años, al punto de ubicarse entre las tres primeras causas de muerte en sujetos de 15 a 44 años, y la segunda en el grupo de 15 a 29 años de edad (López, 2017).De ahí que, se ha señalado que el vacío existencial podría explicar la alta prevalencia de conductas de riesgo en los estudiantes universitarios, que, para el caso de los estudiantes peruanos, implican un consumo excesivo de alcohol, hábitos de alimentación no saludables y conductas sexuales riesgosas (Becerra, 2016). En ese sentido, la falta de orientación vital también se ha vinculado con trastornos alimentarios (Carreras y Duero, 2012), los trastornos de ansiedad (Villanueva y Ugarte, 2017) y el consumo de sustancias psicoactivas (Chau, 1995).

En Arequipa, segunda ciudad del Perú, se ha encontrado que uno de los factores de riesgo en el suicidio de una muestra de 799 personas de entre 6 y 73 años, fue el sexo femenino, presentar problemas familiares y atravesar por trastornos adaptativos (Hancco y Pérez, 2006). En otro estudio, se logró encontrar 
una relación significativa entre el riesgo de suicidio y el locus de control externo, en una muestra de 1023 adolescentes entre 14 y 16 años (Galagarza, 2006). Otra investigación más reciente, reportó que, en una muestra de escolares de dos instituciones públicas de la ciudad, el 12\% aproximadamente, presenta indicios de estar experimentando una crisis existencial con la consecuente neurosis noógena que deviene de una inadecuada respuesta al sentido de vida (Huamani y Ccori, 2016). Y otro estudio ha encontrado que en una muestra de 1225 adolescentes entre 11 y 18 años, provenientes de colegios públicos y privados de la ciudad, poco más del 44\% tiene cumple los criterios psicométricos de sintomatología depresiva (Rivera, Arias y Cahuana, 2017).

En ese sentido, es importante tener presente que las poblaciones de adolescentes son muy vulnerables a tener problemas existenciales, por cuanto tienen que afrontar una crisis de identidad que supone para esta etapa de la vida, formular un proyecto de vida, definir su personalidad, establecer relaciones interpersonales con sus pares del sexo opuesto y tomar decisiones sobre su vida futura (Erikson, 1985/2000). En muchos casos, cuando los apoyos familiares no son los adecuados, los adolescentes no encuentran sentido a su vida presente y futura, se frustran y se desencadena una espiral de conductas violentas que podrían orillarlos a padecer una diversidad de trastornos mentales (Arias, 2013)

Dado este panorama, consideramos que es necesario indagar más sobre el sentido de vida en los adolescentes de la ciudad de Arequipa, desde un enfoque logoterapéutico, sin embargo, en nuestro medio, no se cuenta con instrumentos adecuados para valorar este constructo psicológico. De este modo, aunque las ideas de Frankl han gozado de amplia difusión en el Perú (Amorós, 1996, 2003; Arboccó, 2015; Ramos, 2015), los estudios empíricos sobre el sentido de vida son muy escasos (Huamani, 2017; Huamani y Ccori, 2016). Por ello, el presente estudio, pretende analizar las propiedades psicométricas del Test de Propósito de Vida inspirado en la teoría de Frankl y creado por Crumbaugh \& Maholick, (1969).

Esta prueba consta de tres partes, de las cuales, la primera es de tipo cuantitativo y se compone de 20 ítems en una escala tipo Likert. Inicialmente creada en 1964 (Crumbaugh \& Maholick, 1964), ha sido validada en diversos países y goza de amplia difusión en todo el mundo (Crumbaugh, 1968). Esta prueba presenta coeficientes de confiabilidad muy aceptables, aunque su estructura factorial no siempre ha sido confirmada. En ese sentido, la versión original, solo cuenta con un factor (Crumbaugh \& Maholick, 1969) pero en el estudio de Duffon \& Pearlman (1986) se reportaron dos factores: satisfacción con la vida y propósito de vida, al igual que Morgan \& Farsides (2009) quienes denominaron a sus dos factores hallados como vida apasionante y vida con propósito.

En países de habla hispana, Noblejas (1994) fue una de las primeras autoras en valorar las propiedades psicométricas del Purpose in Life Test en una muestra 
de 823 españoles, determinando una estructura de cuatro factores que explican el $54 \%$ de la varianza total. También ha valorado la fiabilidad del PIL y del Logotest, otra prueba de orientación logoterapéutica (Noblejas, 2000), creada por Elizabeth Lukas (2001) que también evalúa el sentido de vida, y que ha sido objeto de estudios de corte psicométrico en países de habla hispana (Sánchez, Risco y Urchaga, 2011).

En México, se aplicó el Purpose in Life Test a 723 estudiantes universitarios de 23 carreras diferentes y se reportó una estructura de tres factores: Percepción de sentido y significado de la vida, Satisfacción por la propia vida y Miedo a la muerte - Libertad y control de vida; que explican el $50 \%$ de la varianza total, con un índice de confiabilidad de 0.904 obtenido mediante la prueba alfa de Cronbach (Magaña, Zavala, Ibarra, Gómez y Gómez, 2004). En Colombia, Martínez, Trujillo y Trujillo (2012) han reportado una estructura del Purpose in Life Test de tres factores con bajos índices de confiabilidad pero con mayor ajuste estadístico según el análisis factorial confirmatorio, que mantiene los 20 ítems originales.

Otro estudio, también en Colombia, reportó que el PIL tiene una estructura de dos factores, pero se eliminaron 10 ítems con la finalidad de elevar sus valores de confiabilidad. Asimismo, el análisis factorial confirmatorio determinó la presencia de os factores, uno cognitivo evaluativo relacionado con la percepción del sentido de vida y otro motivacional relacionado con el establecimiento de metas (García-Alandete, Martínez y Sellés, 2013). En Argentina, Gottfried (2016) aplicó el PIL test a 1441 personas entre 15 y 79 años de edad, reportando que la prueba se compone de tres factores con niveles adecuados de confiabilidad según el método de consistencia interna con la prueba alfa de Cronbach: Percepción de sentido con 0.83 , Vivencia de sentido con 0.76 y Actitud ante la muerte con 0.66.

De acuerdo a los resultados obtenidos con el PIL, se ha reportado que la mayoría de sujetos evaluados presentan niveles adecuados de sentido de vida (Magaña et al., 2004; García-Alandete et al., 2009; Huamani y Ccori, 2016) y que no existen diferencias entre varones y mujeres, pero sí en cuanto a la edad, pues a partir de los 31 años se ha observado un incremento estadísticamente significativo del sentido de vida (Gottfried, 2016).

Así, el propósito de esta investigación, en analizar las propiedades psicométricas del Purpose in Life Test en una muestra de escolares de la ciudad de Arequipa, por lo tanto, se trata de un estudio instrumental (Montero y León, 2007).

\section{MÉTODO}

\section{Muestra}

La muestra está conformada por 872 estudiantes de cinco instituciones educativas públicas de la ciudad de Arequipa. El 46.9\% son varones y el 53.1\% son mujeres con una edad media de 15 años y una desviación estándar de \pm 0.8 dentro de un 
rango de 14 a 18 años de edad. El 51.8\% fueron del 4to de secundaria y $48.2 \%$ del 5to de secundaria. Las instituciones educativas fueron seleccionadas de manera intencional debido a las facilidades dadas para evaluar a los estudiantes. Se aplicó la técnica de muestreo de grupos intactos para evaluar a todos los estudiantes, quienes fueron informados de los fines del estudio, garantizándoles la confidencialidad de los datos con fines exclusivos de investigación.

\section{Instrumento}

Se aplicó el Purpose in Life Test de Crumbaugh \& Maholick (1969), en su versión validada para población hispanoparlante por Noblejas (1994, 2000). Esta prueba se puede aplicar para personas a partir de los 14 años, ya sea de manera individual o colectiva con una duración entre 15 y 30 minutos. La prueba evalúa el sentido de vida de acuerdo con los criterios de Viktor Frankl (1983, 1975/1987, 1962/2002) y consta de 20 ítems. Noblejas (1994) reportó una estructura factorial de cuatro factores como se muestra líneas abajo:

Percepción de sentido (PS): captación de motivos y razones para vivir la vida y la valoración que está en general merecey se compone de los ítems $4,6,9,10$, $11,12,16,17,20$.

Experiencia de sentido (ESE): percepción de la vida propia y de la vida cotidiana como plenas de cosas buenas, que comprende los ítems 1, 2, 5, 9, 17, $19,20$.

Metas y tareas (MT): objetivos ligados a acciones concretas de la vida y la responsabilidad personal percibida hacia los mismos, y se compone los ítems 3,7 , 8, 13, 17, 19, 20.

Dialéctica/destino y libertad (DDL): tensión destino vs. libertad y afrontamiento de la muerte como acontecimiento incontrolable, impredecible e inevitable, que abarca los ítems 14, 15, 18.

Asimismo, esta autora encontró niveles de confiabilidad adecuados al correlacionar las medidas del PIL con el Logotest al 99.9\% (Noblejas, 2000).

\section{Procedimiento}

Para poder evaluar a los estudiantes se procedió a solicitar los permisos correspondientes ante las autoridades de las instituciones educativas escogidas. Luego se coordinaron las fechas y horarios de evaluación con los profesores encargados de cada salón. Antes de la aplicación se explicaron los fines del estudio y los estudiantes firmaron un consentimiento informado que se adjuntó al protocolo de evaluación de la prueba. De este modo, los estudiantes fueron evaluados colectivamente en sus respectivos salones de clase y con la venia del 
profesor de turno, dentro del horario escolar en los tunos de mañana y tarde. Los datos fueron recogidos durante los meses de agosto y octubre del año 2016.

\section{Análisis de datos}

Para el análisis estadístico se siguieron los procedimientos estándar para investigaciones psicométricas (Livia y Ortiz, 2014).Se aplicó el software SPSS 20.0 (IBM, 2011) para el análisis factorial exploratorio (AFE) y el cálculo de la confiabilidad; además del módulo SPSS AMOS (Arbuckle, 2013) para el análisis factorial confirmatorio (AFC).

\section{RESULTADOS}

\section{Validez}

La validez apunta a estudiar el grado en el que una prueba mide aquello que dice medir (Livia y Ortiz, 2014) y se comprobó analizando los puntajes del Test de Sentido de Vida o Prueba de los Propósitos Vitales a través de la validez de constructo.Los índices de validez alcanzados se aprecian en la Tabla 1:

\section{Tabla 1}

Análisis de componentes principales y correlaciones de las áreas del PIL

\begin{tabular}{lllcccc}
\hline \multicolumn{1}{c}{ ESCALAS } & \multicolumn{1}{c}{ Ítems } & M & DE & Factor & $\begin{array}{c}\text { total de } \\
\text { varianza } \\
\text { explicada }\end{array}$ & r \\
\hline Percepción de sentido & $16,11,10,9,6,12,17,18,19$ & 35.61 & 7.49 & 39.58 & & $.914^{* *}$ \\
Metas y tareas & $3,20,7,4,13,8$ & 12.35 & 2.47 & 5.67 & 56.02 & $.710^{* *}$ \\
Experiencia de sentido & $2,1,5,14$ & 26.87 & 4.61 & 5.6 & $.822^{* *}$ \\
Dialéctica destino/libertad & 15 & 24.64 & 5.42 & 5.17 & $.855^{* *}$ \\
\hline
\end{tabular}

Medida de adecuación al muestreo de Kaiser - Mayer - Olkin $=0.953$

Test de Esfericidad de Bartlett $=6740.87$

$\mathrm{P}<.0001$

Nota: $* * \mathrm{p}<.01$ (bilateral).

El análisis de la validez de constructo realizado a través del análisis factorial exploratorio con base al método de los componentes principales y al método de rotación varimax, presenta un valor de adecuación al muestreo de KaiserMayer-Olkin (KMO) de 0.953 (se requiere un índice mayor a 0.50 ) y ul test de esfericidad de Bartlett que es significativo $(\mathrm{p}<.01)$ lo cual garantiza la pertinencia de la ejecución del análisis factorial exploratorio. Asimismo, todos los indicadores correlacionaron positiva y significativamente con la puntuación total de la escala. Los resultados indican la existencia de cuatrofactores que permiten explicar el $56.02 \%$ de la varianza total (ver Tabla 2). 
Tabla 2

Matriz de componente rotado

\begin{tabular}{|c|c|c|c|c|}
\hline \multirow{2}{*}{ Ítems del PIL } & \multicolumn{4}{|c|}{ Componente } \\
\hline & 1 & 2 & 3 & 4 \\
\hline 16. Con respecto al suicidio, yo: & .756 & & & \\
\hline 11. Al pensar en mi propia vida: & .683 & & & \\
\hline 10. Si muriera hoy me parecería que mi vida ha sido: & .667 & & & \\
\hline 9. Mi vida está: & .634 & & & \\
\hline 6. Si pudiera escoger, usted preferiría: & .562 & & & \\
\hline 12. Al considerar el mundo en relación con mi vida, el mundo: & .526 & & & \\
\hline $\begin{array}{l}\text { 17. Considero que mi capacidad para encontrar un significado, un propósito o una } \\
\text { misión en la vida es: }\end{array}$ & .512 & & & \\
\hline 18. Mi vida está: & .388 & & & \\
\hline 19. El enfrentarme a mis tareas cotidianas constituye: & .372 & & & \\
\hline 3. En la vida tengo: & & .832 & & \\
\hline 20. He descubierto para mi vida: & & 661 & & \\
\hline 7. Después de terminar de estudiar me gustaría: & & .653 & & \\
\hline 4. Mi existencia personal es: & & .653 & & \\
\hline 13. Me considero: & & .434 & & \\
\hline 8. En el logro de mis metas: & & .424 & & \\
\hline 2. La vida me parece: & & & .801 & \\
\hline 1. Generalmente me encuentro: & & & .691 & \\
\hline 5. ¿cada día es? & & & 689 & \\
\hline $\begin{array}{l}\text { 14. Con respecto a la libertad que se dispone para hacer las propias elecciones, creo } \\
\text { que el hombre es: }\end{array}$ & & & .398 & \\
\hline 15. Con respecto a mi muerte, yo: & & & & .846 \\
\hline
\end{tabular}

Los estadísticos descriptivos muestran que ninguno de los indicadores presenta valores elevados de asimetría o curtosis, por lo que las distribuciones no son excesivamente no normales (Tabla 3).

Tabla 3

Test de Kolmogorov de bondad de ajuste a la curva normal del PIL

\begin{tabular}{lcccc}
\hline ESCALAS & M & DE & Asimetría & Curtosis \\
\hline Percepción de sentido & 35.61 & 7.49 & -0.662 & 0.263 \\
Metas y tareas & 12.35 & 2.47 & 0.557 & 0.307 \\
Experiencia de sentido & 26.87 & 4.61 & -0.731 & 0.301 \\
Dialéctica destino/libertad & 24.64 & 5.42 & -0.596 & 0.218 \\
\hline
\end{tabular}




\section{Confiabilidad}

La confiabilidad se analizó mediante el métododeconsistencia interna con la prueba alfa de Cronbachpara cada uno de los cuatro factores del test. En la Tabla 4 se presenta el análisis de la confiabilidad de los factores y el test de sentido de vida completo, obteniéndose coeficientes de confiabilidad superiores al criterio de 0.70 (Brown, 1970/1980). El coeficiente de confiabilidad del test completo fue de 0.91 considerado como bueno y que nos indica que el test de sentido de vida es una medida confiable.

Tabla 4

Análisis de la confiabilidad del PIL

\begin{tabular}{lc}
\hline Factores & Alfa de Cronbach \\
\hline Percepción de sentido & 0.886 \\
Metas y tareas & 0.822 \\
Experiencia de sentido & 0.721 \\
Dialéctica destino/libertad & 0.713 \\
Puntaje total & 0.911 \\
\hline
\end{tabular}

\section{Análisis factorial confirmatorio (AFC)}

El análisis preliminar de los datos muestra que todos los ítems se pueden emplear en el AFC, pues no presentan valores muy elevados de curtosis y asimetría (Tabla 3). Para refinar el análisis factorial de la prueba y construir el modelo de ecuaciones estructurales que confirme los factores (variable latente) a través de las variables observadas (ítems del test), se empleó el AMOS v. 23 de IBM.

Una vez obtenidos los factores, producto de la base teórica (construcción del test) realizado por Noblejas $(1994,2000)$ y el análisis factorial exploratorio mediante SPSS v.23, se construyó con el AMOS el modelo análogo para cada estructura factorial (variable latente), con el fin de valorar si presentan buenos índices de bondad de ajuste. Además, se calcularon los índices de modificación para correlacionar (covarianzas) aquellos errores de las variables observadas (items) de una misma variable latente (factores) que presenten valores inusuales.

Este test de sentido de vida confirma el modelo teórico propuesto por los autores, pues los 20 ítems (variables observables), confirman cuatro factores o variables latentes. Este análisis factorial confirmatorio (AFC), mejora el ajuste y elimina dos ítems: el 4 y el 19.Además, correlaciona el ítem 6 (de la variable latente Percepción de sentido PS) con el ítem 13 (de la variable latente Metas y tareas MT), información que nos indica que probablemente ambas variables observables estén midiendo lo mismo o que generen una nueva variable latente. Lo mismo sucede con el ítem 20 y el ítem 3 de las variables latentes Experiencia 
de sentido (ESE) y la variable Metas y tareas (MT), respectivamente. Cabe mencionar que el ítem 20, fue considerado como una variable observable en tres de los factores en el modelo inicial propuesto por los autores. Asimismo, se comprueba el AFC, porque los índices de bondad de ajuste cumplen con los requisitos que se exigen.

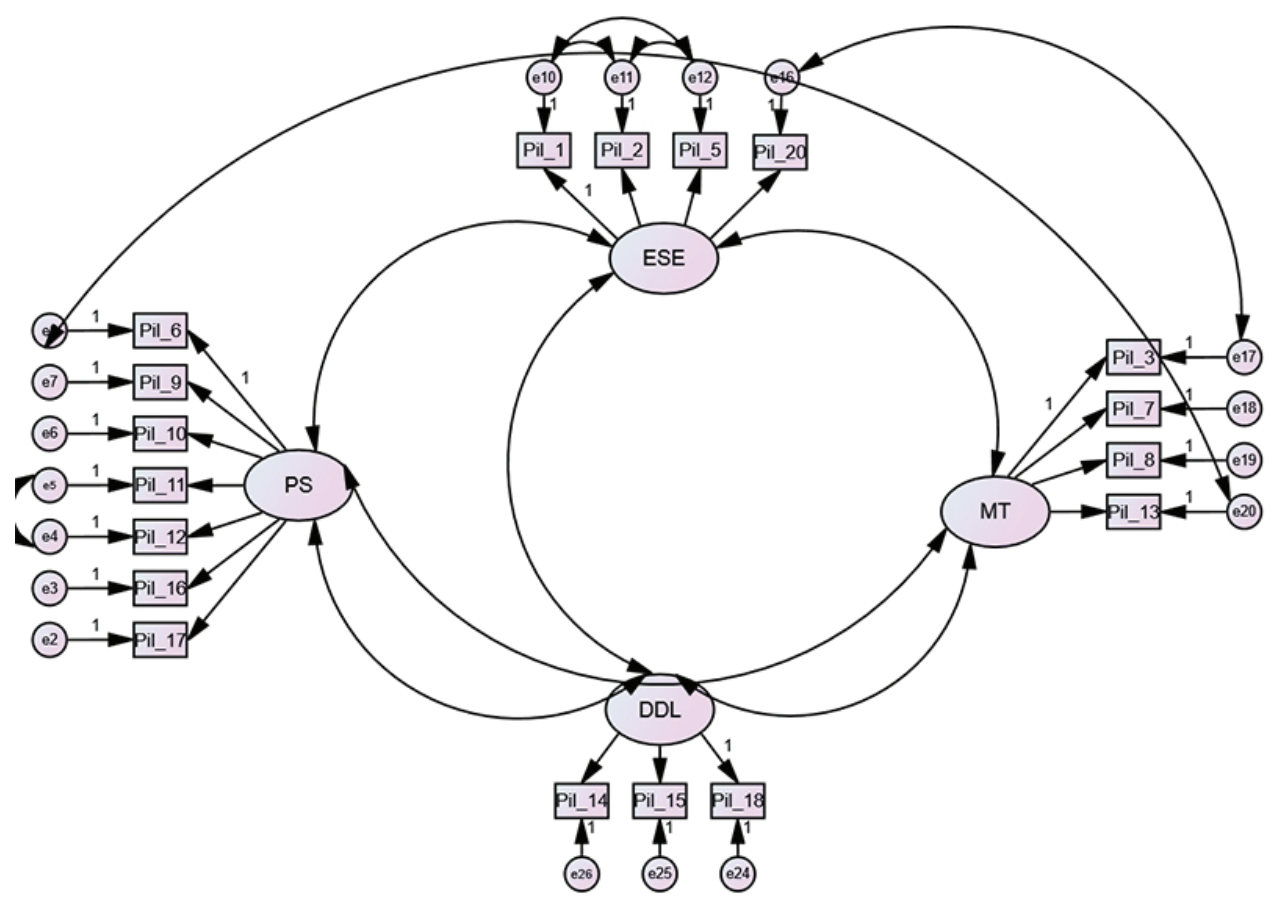

Figura 2. Modelo final estimado del test de sentido de vida (PIL)

Con los factores obtenidos (variables latentes) y en base a la teoría de Noblejas (2000), se evaluó el modelo final con todos los ítems (variables observables) y errores, para todas las relaciones causales y correlaciones del modelo. La p-valor asociada a la estimación significa que $p$ es la probabilidad de que el valor estimado no sea distinto de 0 en realidad.

Después de realizar el análisis factorial confirmatorio, los ítems que conforman la variable sentido de vida son los que se presentan en la Tabla 6. En consecuencia, del modelo de cuatro factores no fue desechado ningún factor, pero sí se eliminaron los ítems 4 y 19, debido a que no discriminan el factor, ni cumplen con los parámetros de la ecuación estandarizada. 
Tabla 5

Indicadores de bondad de ajuste del AFC delPIL

\begin{tabular}{lccc}
\hline \multicolumn{1}{c}{ Estadístico } & Abreviatura & Criterio & Resultado \\
\hline Ajuste absoluto & & & \\
Chi-cuadrado & $\chi 2$ & Significación $>.05$ & \\
Razón Chi-cuadrado / grados de libertad & $\chi 2 / \mathrm{gl}$ & Menor que 3 & 2.762 \\
\hline Ajuste comparativo & & & \\
$\quad$ Índice de bondad de ajuste comparativo & CFI & $\geq 0.90$ & 0.961 \\
$\quad$ Índice de ajuste normalizado & NFI & $\geq 0.95$ & 0.951 \\
\hline Ajuste parsimonioso & & & \\
$\quad$ NFI corregido por parsimonia & PNFI & Próximo a 1 & 0.756 \\
\hline Otros & & & \\
$\quad$ Índice de bondad de ajuste & GFI & $\geq 0.80 \geq 0.9$ & 0.958 \\
Raíz del residuo cuadrático promedio de aproximación & RMSEA & $<0.08$ & 0.045 \\
\hline
\end{tabular}

Tabla 6

AFC realizado hasta obtener la solución terminaldel PIL

\begin{tabular}{lll}
\hline Variable & Factores & Ítems \\
\hline & Percepción de sentido (PS) & $6,9,10,11,12,16,17$ \\
Sentido de vida (PIL) & Experiencia de sentido (ESE) & $1,2,5,20$ \\
& Metas y tareas (MT) & $3,7,8,13$ \\
& Dialéctica destino/libertad (DDL) & $14,15,18$ \\
\hline
\end{tabular}

\section{DISCUSIÓN}

La prueba de sentido de vida como se le conoce en español al Purpose in Life Test de Crumbaugh \& Maholick (1964, 1969), ha sido uno de los instrumentos más utilizados para valorar el constructo correspondiente en distintas muestras de sujetos de Iberoamérica (García-Alandete, Gallego-Pérez y Pérez-Delgado, 2009; Huamani, 2017; Huamani y Ccori, 2016; Martínez y Castellanos, 2013). Ello ha supuesto una diversidad de estudios psicométricos en países como España, Colombia, México, Argentina, etc. (García-Alandete, Martínez y Sellés, 2013; Gottfried, 2016; Magaña et al., 2004; Martínez, García-Alandete, Sellés, Bernabé y Soucase, 2012; Martínez, Trujillo y Trujillo, 2012; Noblejas, 1994, 2000) que dan cuenta de sus propiedades de validez y confiabilidad.

En el Perú, sin embargo, no se han hecho estudios psicométricos con el PIL, mucho menos en la ciudad de Arequipa. En tal sentido, el presente artículo se planteó como objetivo analizar las propiedades psicométricas del Purpose in Life Test en una muestra de 872 estudiantes de nivel secundario de la ciudad de 
Arequipa. Los resultados difieren de autores como Duffon y Pearlman (1986) o Morgan y Farsides (2009), que reportaron dos factores subyacentes a la prueba; o los de Magaña, Zavala, Ibarra, Gómez y Gómez (2004), Martínez, Trujillo y Trujillo (2012), García-Alandete, Martínez y Sellés, 2013, Gottfried (2016), en muestras latinoamericanas que dan cuenta de tres factores; y más bien confirman los datos de Noblejas $(1994,2000)$ que ha encontrado cuatro factores mediante métodos estadísticos factoriales.

Una consideración importante, es la eliminación de los ítems 4 y 19, por no discriminar su ubicación en un solo factor, por lo cual, se prefirió retirarlos. Este tipo de reducción de ítems también ha sido reportado por otros autores como García-Alandete, Martínez y Sellés (2013), que llegaron a retirar hasta 10 ítems para constituir una versión breve de la prueba de sentido de vida para población colombiana. En ese sentido, es preferible eliminar los ítems que no tienen una saturación clara en uno u otro factor, para elevar los niveles de confiabilidad de la prueba (Livia y Ortiz, 2014). Así, tenemos que mientras el estudio de Martínez, Trujillo y Trujillo (2012) presenta bajos niveles de confiabilidad, pero mantiene los 20 ítems originales, nuestros resultados han encontrado altos niveles de confiabilidad que van de 0.713 hasta 0.911 , para el cuarto factor y la puntuación total, respectivamente.

Por otro lado, los factores que se han confirmado mediante el análisis factorial confirmatorio, corresponden a la estructura reportada por Noblejas (2000), aunque con ligeras modificaciones, en la constitución de los ítems que la conforman. En ese sentido, cabe señalar que mientras los ítems 9, 17, 19, 20 que componen en el modelo tetrafactorial de Noblejas (1994) saturan en dos o hasta tres factores, para nuestro caso hemos preferido eliminar aquellos que tienen una saturación ambigua, como lo han sugerido Martínez, García-Alandete, Sellés, Bernabé y Soucase (2012).

Los factores que hemos podido confirmar son Percepción de sentido, Experiencias de sentido, Metas y tareas, y Dialéctica destino/libertad; que corresponden a características que se encuentran íntimamente ligadas a la autorrealización y tendencias productivas (Maslow, 1968); y que pueden sumarse a la búsqueda de superación personal, la perseverancia, el proyecto de vida, la autoestima, las relaciones interpersonales profundas, los valores morales y la creatividad (Magaña et al., 2004), como tendencias que adquieren relevancia en la dotación de sentido que se cultiva cuando el ser humano está bien ubicado en el aquí y el ahora, cuando sus acciones están orientadas hacia la trascendencia y la autorrealización, a través del desarrollo de sus fortalezas que se dirigen y canalizan hacia objetivos o metas que se forjan en el curso del desarrollo humano, sobre la base del autoconocimiento y una concepción optimista del mundo (Frankl, 1994). 
En cuanto a las limitaciones metodológicas, podemos incluir el muestreo no probabilístico y la imposibilidad de generalizar los datos a toda la población escolar de la ciudad de Arequipa. Sin embargo, dado que este es el primer estudio de corte psicométrico que se realiza con el PIL en esta ciudad, podemos señalar que facilitará su uso en estudios con muestras de estudiantes de nivel secundario, donde se han evidenciado diversos factores de riesgo psicosocial que actúan como catalizadores de la crisis existencial que afecta a los jóvenes, y que se refleja a través de los altos índices de suicidio en menores de 30 años, el incremento de casos de depresión en población infantil y adolescente, así como la falta de

De este modo, el PIL, viene a sumarse a una amplia gama de instrumentos que se utilizan en Latinoamérica (Núñez-Rodríguez, Lazaretti, y Koller, 2016; Suárez-Pinto, Blanco-Gómez, y Díaz-Martínez, 2016) para evidenciar diversos aspectos del vacío existencial que vivencian los niños y jóvenes de las generaciones presentes. En ese sentido, es importante que más instrumentos psicológicos sean adaptados o validados a la realidad peruana, sobre todo en muestras del interior del país, ya que existe una gran heterogeneidad cultural entre las personas que habitan las diferentes regiones que componen el territorio peruano.

\section{REFERENCIAS BIBLIOGRÁFICAS}

Adler, A. (1914/1961). Psicología del individuo. Colección Biblioteca del Hombre Contemporáneo, vol 79. Buenos Aires: Editorial Paidós

Amorós, V. (1996). Psicología y psicoterapia humanista. Revista de Psicología (Pontificia Universidad Católica del Perú),14(1), 73-80.

Amorós, V. (2003). Psicoterapia humanista. Revista de Psicología (Universidad Nacional Mayor de San Marcos), 4(1), 171-180.

Arboccó, M. (2015). Psicología, psicoterapia y sociedad: Reflexiones humanístico existenciales. Avances en Psicología, 23(1), 49-60.

Arbuckle, J. (2013). IBM SPSS Amos 21 User's Guide. USA: IBM.

Arias, W. L. (2013). Agresión y violencia en la adolescencia: la importancia de la familia. Avances en Psicología, 21(1), 23-34.

Becerra, S. (2016). Descripciones de las conductas de salud en un grupo de estudiantes universitarios de Lima. Revista de Psicología (PontificaUniversidad Católica del Perú), 34(2), 239-260.

Brown, F. G. (1970/1980). Principios de la medición en psicología y educación. México: Manual Moderno.

Carreras, X., y Duero, D. G. (2012). Sentimiento de orientación vital y vivencia del cuerpo en personas con trastornos de la Alimentación: Un estudio fenomenológico-narrativo. Revista Argentina de Ciencias del Comportamiento, 4(3), 30-47. 
Ceballos, F., y Chávez, A. (2016). Perfil de suicidas chilenos vía análisis de contenido de notas póstumas. Avances en Psicología Latinoamericana, 34(3). 517-528.

Chau, C. (1995). Factores psicosociales asociados al uso de bebidas alcohólicas y tabaco en adolescentes tardíos de una universidad privada de Lima. Revista de Psicología (Pontificia Universidad Católica del Perú), 13(2), 161-191.

Crumbaugh, J. (1968). Cross-validation of Purpose in Life test based on Frankl's concepts. Journal of Individual Psychology, 24, 74-81.

Crumbaugh, J., \& Maholick, L. (1964). An experimental study in existentialism. The psychometric approach to Frankl's concept of noogenic neurosis. Journal of Clinical Psychology, 20,200-207.

Crumbaugh, J., \& Maholick, L. (1969). Manual of instruction for the Purpose in Life Test. Saratoga: Viktor Frankl Institute.

Duffon, B. D., \& Pearlman, D. (1986). The association between religiosity and the purpose in life test: Does it reflect purpose or satisfaction? Journal of Psychology and Theology, 14, 42-48

Edwards, M. J., \& Holden, R. R. (2003). Coping, meaning in life, and suicidal manifestations: Examining gender differences. Journal of ClinicalPsychology, 59(10), 1133-1150.

Ellis, A. (1962/1980). Razón y emoción en psicoterapia. Bilbao: Descleé de Brouwer.

Erikson, E. H. (1985/2000). El ciclo vital completado. Barcelona: Paidós.

Frankl, V. (1980). Ante el vacío existencial. Hacia una humanización de la psicoterapia. Barcelona: Herder.

Frankl, V. (1946/1983). El hombre en busca de sentido. Barcelona: Herder.

Frankl. V. (1975/1987). El hombre doliente. Fundamentos antropológicos de la psicoterapia. Barcelona: Herder.

Frankl, V. (1994). Logoterapia y análisis existencial. Barcelona: Herder.

Frankl. V. (1962/2002). La voluntad de sentido: Conferencias escogidas sobre logoterapia. $3^{\text {th }}$ ed. Barcelona: Herder.

Frankl, V. (1985/2006). La presencia ignorada de Dios. 12 $2^{\text {th }}$ ed. Barcelona: Herder.

Galagarza, L. (2006). Locus de control y riesgo suicida en adolescentes. Revista de Psicología (Universidad Católica de Santa María), 3, 37-43.

Gallego, J., y García, J. (2004). Sentido en la vida y desesperanza en un grupo de estudiantes universitarios. NOUS.Boletín de Logoterapia y Análisis Existencial, 8, 49-64.

García, L. (1999). Configuración histórica del concepto de autoimagen. Revista de Historia de la Psicología, 20(3-4), 405-414.

García-Alandete, J., Gallego-Pérez, J., y Pérez-Delgado, E. (2009). Sentido de la vida y desesperanza: un estudio empírico. UniversitasPsychologica, 8, 447-454. 
García-Alandete, J., Martínez, E., y Sellés, P. (2013). Estructura factorial y consistencia interna de una versión española del Purpose-In-Life Test. UniversitasPsychologica, $12(2), 517-530$.

Gottfried, A. E. (2016). Adaptación argentina del PIL test (Test de Sentido de Vida) de Crumbaugh y Maholick. Revista de Psicología (Universidad Católica Argentina), 12(23), 49-65.

Hancco, L. D., y Pérez, A. A. (2006). Factores de riesgo del intento de suicidio. Revista de Psicología (Universidad Católica de Santa María), 3, 44-53.

Huamani, J. C. (2017). Sentido de vida en familiares cuidadores de personas diagnosticadas con cáncer. Avances en Psicología, 25(1), 97-106.

Huamani, J. C., y Ccori, J. (2016). Respuesta al sentido de vida. Revista de Psicología de Arequipa, 6(1), 331-348.

IBM. (2011). IBM SPSS Statistics (Version 20) [programa informático en CD-ROM].

James, W. (1890/1996). Principios de Psicología. México: Fondo de CulturaEconómica.

Jaspers, K. (1913/1996). Psicopatología general. México: Fondo de CulturaEconómica.

Jiménez, J. M. (2004). Sentidos de vida: razón, emoción y salud desde una perspectiva antropológica. Revista Cubana de Psicología, 21(2), 129-134.

Kelly, G. (1955/1966). Teoría de la personalidad. Buenos Aires: Ediciones Troquel.

Livia, J., \& Ortiz, M. (2014). Construcción de pruebas psicométricas: Aplicaciones a las ciencias sociales y de la salud. Lima: Universidad Nacional Federico Villarreal.

López, L. C. (2007). Caracterización de riesgo diferencial-demográfico en urgencias psicológicas: intento de suicidio y otros motivos de consulta. Revista Argentina de Ciencias del Comportamiento, 9(1), 44-53.

Lukas, E. (2001). Paz vital, plenitud y placer de vivir. Los valores de la logoterapia. Barcelona: Paidós.

Magaña, L., Zavala, M. A., Ibarra, I., Gómez, M. T., y Gómez, M. M. (2004). El sentido de vida en estudiantes de primer semestre de la Universidad de la Salle Bajío. Revista del Centro de Investigación de la Universidad La Salle, 6(22), 5-13.

Martínez, E., Trujillo, A. M., y Trujillo, C. A. (2012). Validación del test de Propósito Vital (PIL Test - Purpose in Life Test) para Colombia. Revista Argentina de Clínica Psicológica, 21(1), 85-93.

Martínez, E., y Castellanos, C. (2013). Percepción del sentido de vida en universitarios colombianos. Pensamiento Psicológico,11(1), 71-82.

Martínez, E., García-Alandete, J., Sellés, P., Bernabé, G., y Soucase, B. (2012). Análisis factorial confirmatorio de los principales modelos propuestos para el Purpose-inLife Test en una muestra de universitarios españoles. ActaColombiana de Psicología, 15(1), 67-76. 
Maslow, A. H. (1968). Toward a psychology of being. USA: Insight Book.

Medina, A. R., Herazo, M. I., Barrios, A. M., Rodelo, Y. V., y Salinas, L. F. (2017). Factores psicosociales asociados a la conducta suicida en adolescentes. Avances en Psicología, 25(1), 49-57.

Montero, I., \& León, O. G. (2007). A guide for naming research studies in Psychology. International Journal of Clinical and Health Psychology, 7(3), 847-862.

Morgan, J., \& Farsides, T. (2009). Measuring meaning in life. Journal of Happiness Studies, 10, 197-214.

Noblejas, M. A. (1994). Logoterapia. Fundamentos, principios y aplicación. Una experiencia de evaluación del "logro interior de sentido". (Tesis Doctoral). Universidad Complutense de Madrid, España.

Noblejas, M. A. (2000). Fiabilidad de los tests PIL y Logotest. Nous.Boletín de Logoterapia y Análisis Existencial, 4, 81-90.

Núñez-Rodríguez, S., Lazaretti, A. P., \& Koller, S. H. (2016). Cross-cultural adaptation and psychometric properties of the Portuguese version of the Aspiration Index (AI). Trends in Psychology / Temas emPsicologia, 24(3), 1169-1180.

Pareja, L. G. (2007). El mundo y el tiempo de Viktor Frankl. Buenos Aires: San Pablo.

Pinedo, I. A. (2014). Apropiación del sufrimiento y búsqueda del sentido. Tesis Psicológica, 9(1), 36-49.

Quiceno, J. M. y Vinaccia, S. (2009). La salud en el marco de la psicología de la religión y la espiritualidad. Diversitas, 5(2), 321-336.

Ramos, L. A. (2015). Una aproximación al significado de análisis existencial en la logoterapia de Víktor Frankl. Avances en Psicología, 23(2), 149-154.

Rey de Castro, J. (2011). Aportes antropológicos de la aproximación de Heráclito al alma humana. Revista de Investigación, 2, 99-167.

Rivera, R., Arias, W. L., y Cahuana, M. (2017). Perfil familiar de adolescentes con sintomatología depresiva en la ciudad de Arequipa, Perú. Revista Chilena de Neuropsiquiatría. (artículo sometido a revisión).

Sánchez, A., Risco, A., y Urchaga, J. D. (2011). Propiedades psicométricas de un instrumento (Logotest) para evaluar el sentido de vida. International Journal of Developmental and Educational Psychology, 5(1), 373-381.

Suárez-Pinto, T. A., Blanco-Gómez, A., y Díaz-Martínez, L. A. (2016). Validación de la versión en español del Rapid AssessmentforAdolescentPreventiveServices en adolescentes colombianos. Archivos Argentinos de Pediatría, 114(5), 448-457.

Villanueva, L. E., y Ugarte, A. R. (2017). Niveles de ansiedad y la calidad de vida en estudiantes de una universidad privada de Arequipa. Avances en Psicología, 25(2), 153-169.

Yalom, I. D. (1980). Existential Psychotherapy. USA: BasicBooks. 